\title{
Connecting certain rigid birational non-homeomorphic Calabi-Yau threefolds via Hilbert scheme
}

\author{
Nam-Hoon LeE AND KeIJI Oguiso
}

\begin{abstract}
We shall give an explicit pair of birational projective Calabi-Yau threefolds which are rigid, non-homeomorphic, but are connected by projective flat deformation over some connected base scheme.
\end{abstract}

\section{Introduction}

A Calabi-Yau manifold is a compact Kähler simply connected manifold with a nowhere vanishing global $n$-form but no global $i$-form with $0<i<n=$ $\operatorname{dim} X$. By Kodaira's criterion, it is projective if the dimension $n \geq 3$.

As well known, Calabi-Yau manifolds, hyperkähler manifolds and complex tori form the building blocks of compact Kähler manifolds with vanishing first Chern class [2,6]. A famous theorem of Huybrechts states that two bimeromorphic hyperkähler manifolds are equivalent under smooth deformation $[8,9]$. In particular, they are homeomorphic to each other, having the same Betti numbers and Hodge numbers. Clearly, the same holds true for complex tori. Another theorem, originally due to Batyrev and Kontsevich, says that two birational Calabi-Yau manifolds have the same Betti numbers and Hodge numbers $[1,4,10,19,21]$. However, there are rigid birational non-isomorphic Calabi-Yau manifolds (cf. Theorem 0.1). Obviously, they are not equivalent under any smooth deformation.

The aim of this paper is to remark that there nevertheless exist birational Calabi-Yau threefolds which are rigid, non-homeomorphic, but are connected by (necessarily non-smooth) projective flat deformation:

Theorem 0.1. There are Calabi-Yau threefolds $X$ and $Y$ such that:

(1) $X$ and $Y$ are birational and rigid,

(2) $X$ and $Y$ are not homeomorphic but, 
(3) $X$ and $Y$ are connected by projective flat deformation over some connected scheme.

Note that any family that connects $X$ and $Y$ necessarily involves very singular spaces (see the toy example in Section 1). This result is experimental but we believe that it is the first attempt to study "deformation" of rigid Calabi-Yau threefolds. This work is also motivated by the first named author's recent result on the equivalence of certain Calabi-Yau threefolds with Picard number one, of different topological type, under projective flat deformation [11].

In the proof of our main theorem (Theorem 0.1), the following deep theorem of Hartshorne [7] (see also [16]) plays an important role:

Theorem 0.2 (Hartshorne). The Hilbert scheme $\operatorname{Hilb}_{\mathbf{P}^{N}}^{P(x)}$ of $\mathbf{P}^{N}$ with fixed Hilbert polynomial $P(x)$ is connected.

So, if two varieties belong to the same Hilbert scheme $\operatorname{Hilb}_{\mathbf{P}^{N}} P(x)$, then they appear as fibers of the universal family $u: \mathcal{U} \longrightarrow \operatorname{Hilb}_{\mathbf{P}^{N}}^{P(x)}$, in which $\operatorname{Hilb}_{\mathbf{P}^{N}}^{P(x)}$ is connected. In this way, they are connected by projective flat deformation.

Let $Z$ be a Calabi-Yau threefold and let $H$ be an ample divisor on $Z$. Then, by the Kodaira vanishing theorem and the Riemann-Roch formula, we have

$$
\operatorname{dim} H^{0}\left(\mathcal{O}_{Z}(n H)\right)=\chi\left(\mathcal{O}_{Z}(n H)\right)=\frac{H^{3}}{6} n^{3}+\frac{H \cdot c_{2}(Z)}{12} n
$$

Here $c_{2}(Z)=c_{2}\left(T_{Z}\right)$ is the second Chern class of $Z$. It is also known that $10 H$ is always very ample on $Z$ [14]. Therefore, as a special case of Theorem 0.2 , one obtains the following:

Theorem 0.3. Two Calabi-Yau threefolds are embedded into a projective space with the same Hilbert polynomial, accordingly belong to the same Hilbert scheme of that projective space and connected by projective flat deformation, if and only if they have ample divisors that have the same values of

$$
H^{3} \text { and } H \cdot c_{2} \text {. }
$$

In general, two Calabi-Yau threefolds are unlikely to be connected by projective flat deformation, especially if they are of different topological type. Let $X$ and $Y$ be a complete intersection of two cubics in $\mathbf{P}^{5}$ and a 
quintic hypersurface in $\mathbf{P}^{4}$, respectively. Then we always have

$$
9 k^{3}=\left(k H_{X}\right)^{3} \neq\left(l H_{Y}\right)^{3}=5 l^{3}
$$

for any positive integers $k, l$, where $H_{X}$ and $H_{Y}$ are the ample generators of the Picard groups of $X$ and $Y$, respectively. So $X$ and $Y$ cannot be connected by any projective flat deformation.

Our Calabi-Yau threefolds in Theorem 0.1 are the famous rigid CalabiYau threefold $X_{\phi}$ constructed by Beauville [3] and its birational modification $X_{T}$ studied by the second named author [13] (see also Section 2).

Our proof (for connectedness) is implicit. So the following question might be interesting:

Question 0.1. Can one describe how $X$ and $Y$ in Theorem 0.1 are connected in more explicit manner?

One can find some relevant work in [20].

The structure of this paper is as follows: we discuss some toy case of elliptic curves in Section 1. This explains some idea behind our consideration. In Section 2, we recall Beauville's rigid Calabi-Yau threefold $X_{\phi}$ and its birational modification $X_{T}$. Sections 3 and 4 are devoted to the proof of Theorem 0.1 .

\section{Toy example: connecting two elliptic curves in two ways}

Let $C_{\lambda}(\lambda \neq 0,1)$ be the elliptic curve defined by the Weierstrass equation

$$
y^{2}=x(x-1)(x-\lambda)
$$

Obviously, any two elliptic curves $C_{\lambda_{1}}$ and $C_{\lambda_{2}}$ are connected by the following projective smooth family:

$$
\begin{aligned}
\psi: \mathcal{X}= & \left\{\left(\left[x_{0}: x_{1}: x_{2}\right], \lambda\right) \in \mathbf{P}^{2} \times \mathcal{B} \mid x_{1}^{2} x_{2}\right. \\
& \left.-x_{0}\left(x_{0}-x_{2}\right)\left(x_{0}-\lambda x_{2}\right)=0\right\} \longrightarrow \mathcal{B}
\end{aligned}
$$

Here and hereafter, we put $\mathcal{B}=\mathbf{P}^{1} \backslash\{0,1, \infty\}$.

Yet, we can connect $C_{\lambda_{1}}$ and $C_{\lambda_{2}}$ by another way.

Let $D$ be a hyperelliptic curve with a hyperelliptic involution $\iota$ and let $\Xi$ be the set of the branch points of $\iota$ in $D /\langle\iota\rangle \simeq \mathbf{P}^{1}$. We consider the natural 
morphisms,

$$
\varphi_{1}: C_{\lambda_{1}} \times \widetilde{D /\langle(-1, \iota)\rangle \longrightarrow D /\langle\iota\rangle} \simeq \mathbf{P}^{1}
$$

and

$$
\varphi_{2}: C_{\lambda_{2}} \times \widetilde{D /\langle(-1, \iota)\rangle \longrightarrow D /\langle\iota\rangle} \simeq \mathbf{P}^{1} .
$$

Here ${ }^{\sim}$ 's are the minimal resolutions. We regard $\varphi_{1}$ and $\varphi_{2}$ as projective flat deformations. Then, for $q \in \Xi$, the scheme-theoretic fiber $\varphi_{1}^{-1}(q)=2 l+$ $l_{0}+l_{1}+l_{\infty}+l_{\lambda_{1}}$ consists of $5 \mathbf{P}^{1}$ 's, intersecting like

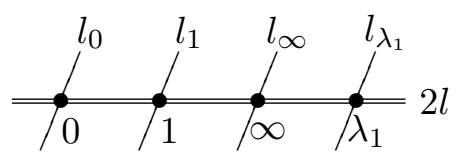

and $\varphi_{1}^{-1}(p) \simeq C_{\lambda_{1}}$ for $p \notin \Xi$. Similarly, the scheme-theoretic fiber $\varphi_{2}^{-1}(q)$ for $q \in \Xi$ is like

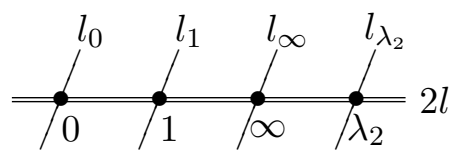

and $\varphi_{2}^{-1}(p) \simeq C_{\lambda_{2}}$ for $p \notin \Xi$. The singular schemes $\varphi_{1}^{-1}(q)$ and $\varphi_{2}^{-1}(q)$ can be put into a projective flat family, in which the fibers are of the form

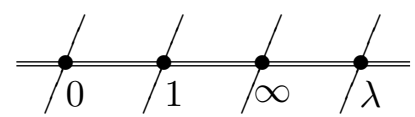

For example, the natural projection $\psi: \mathcal{Y} \longrightarrow \mathcal{B}$, where

$\mathcal{Y}=\left\{\left(\left[x_{0}: x_{1}\right],\left[y_{0}: y_{1}\right], \lambda\right) \in \mathbf{P}^{1} \times \mathbf{P}^{1} \times \mathcal{B} \mid x_{0}^{2} y_{0} y_{1}\left(y_{0}-y_{1}\right)\left(y_{0}-\lambda_{1} y_{1}\right)=0\right\}$

is such a family. In this way, $C_{\lambda_{1}}$ and $C_{\lambda_{2}}$ are connected by a chain of three projective flat deformations.

In the second method, smooth fibers in families are only $C_{\lambda_{1}}$ and $C_{\lambda_{2}}$ and they are connected through very singular spaces. So, the method suggests some possibilities to connect two rigid manifolds of different topological structure. This is the idea behind our construction.

\section{Beauville's rigid Calabi-Yau threefold and its modification}

We briefly recall the two rigid Calabi-Yau threefolds $X$ and $Y$ that appear in Theorem 0.1. 
Let $\zeta=\mathrm{e}^{2 \pi \sqrt{-1} / 3}$. By $E_{\zeta}$, we denote the elliptic curve whose period is $\zeta$ and by $E_{\zeta}^{n} /\langle\zeta\rangle$ the quotient of the $n$-fold product, $E_{\zeta}^{n}$ by the scalar multiplication by $\zeta$. Let

$$
Q_{0}=0, \quad Q_{1}=(1-\zeta) / 3 \quad \text { and } \quad Q_{2}=-(1-\zeta) / 3
$$

in $E_{\zeta}$. These are exactly the fixed points of the scalar multiplication by $\zeta$ on $E_{\zeta}$. For $i_{k}=0,1,2$, let

$$
Q_{i_{1} i_{2} \cdots i_{n}}=\left(Q_{i_{1}}, Q_{i_{2}}, \ldots, Q_{i_{n}}\right) \in E_{\zeta}^{n}
$$

and let $\bar{Q}_{i_{1} i_{2} \ldots i_{n}}$ be its image in $E_{\zeta}^{n} /\langle\zeta\rangle$. Then $\bar{X}=E_{\zeta}^{3} /\langle\zeta\rangle$ has singularities of type $\frac{1}{3}(1,1,1)$ at $\bar{Q}_{i j k}$ 's and the blow-up $\pi: X_{\varphi} \longrightarrow \bar{X}$ at these 27 singular points gives a Calabi-Yau threefold $X_{\phi}$. This is the famous rigid Calabi-Yau threefold found by Beauville [3]. We denote by $E_{i j k}$ the exceptional divisor lying over $\bar{Q}_{i j k}$. The surfaces $E_{i j k}$ is isomorphic to $\mathbf{P}^{2}$.

Let

$$
p_{\phi}: X_{\phi} \longrightarrow B:=E_{\zeta}^{2} /\langle\zeta\rangle
$$

be the morphism, induced by the projection $\operatorname{pr}_{12}: E_{\zeta}^{3} \longrightarrow E_{\zeta}^{2}$. Then we have

$$
p_{\phi}^{-1} \bar{Q}_{i j}=l_{i j} \cup E_{i j 0} \cup E_{i j 1} \cup E_{i j 3} .
$$

Here $l_{i j}$ is a smooth rational curve meeting $E_{i j k}$ transversally. See figure 1. The normal bundle of $l_{i j}$ in $X_{\phi}$ is

$$
N_{X_{\phi} \mid l_{i j}}=\mathcal{O}_{l_{i j}}(-1)^{\oplus 2} .
$$

Performing the elementary transformation along $\bigcup_{i, j} l_{i j}$, we obtain a smooth threefold $X_{T}$. This $X_{T}$ corresponds to that in [13] for $T=\{(i, j) \mid i, j=$ $0,1,2\}$. Denote the proper transform of $E_{i j k}$ in $X_{T}$ by $F_{i j k}$. Note that $F_{i j k}$ is the first Hirzebruch surface $\mathbf{F}_{1}$. Compare figure 2 with figure 1.

Now we summarize some properties of $X_{\phi}$ and $X_{T}$, showed in $[3,13]$.

Theorem 2.1. (1) $X_{\phi}$ and $X_{T}$ are both Calabi-Yau threefolds.

(2) $h^{1,2}\left(X_{\phi}\right)=h^{1,2}\left(X_{T}\right)=0$, i.e., $X_{\phi}$ and $X_{T}$ are rigid.

So, $X_{\phi}$ and $X_{T}$ are birational, rigid Calabi-Yau threefolds. In fact, these $X_{\phi}$ and $X_{T}$ are the Calabi-Yau threefolds $X$ and $Y$ in our Theorem 0.1. We shall show that $X_{\phi}$ and $X_{T}$ are non-homeomorphic in Section 3 and that $X_{\phi}$ and $X_{T}$ are connected by projective flat deformation in Section 4 . 

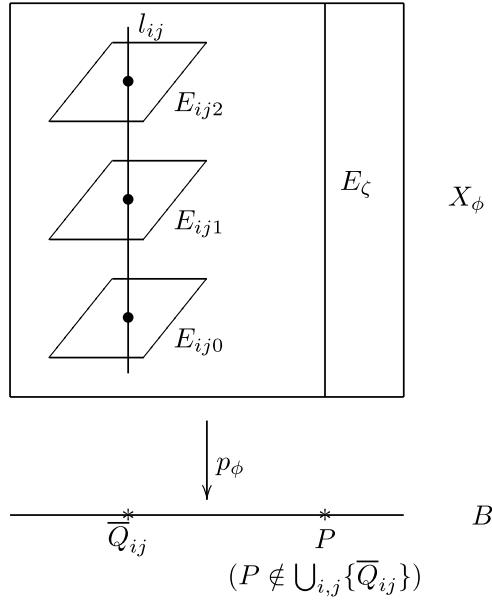

Figure 1: $p_{\phi}: X_{\phi} \rightarrow B$

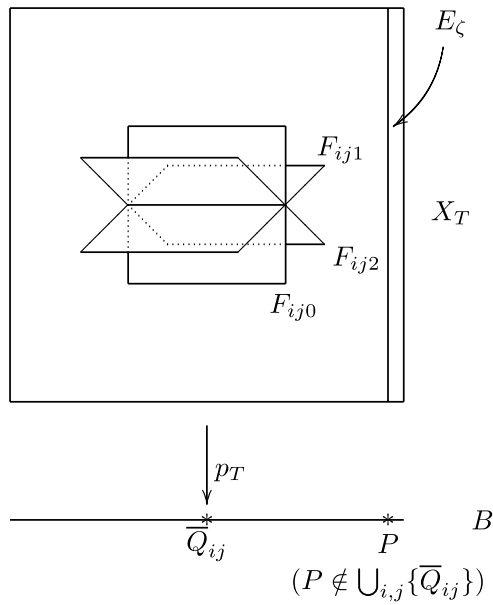

Figure 2: $p_{T}: X_{T} \rightarrow B$

Here, we summarize notations which will be frequently used in the next two sections:

\section{Notation 2.1.}

- $\zeta=e^{2 \pi \sqrt{-1} / 3}$, the primitive third root of unity in the upper half plane.

- $E_{\zeta}=\mathbf{C} /(\mathbf{Z} \oplus \mathbf{Z} \zeta)$ is the elliptic curve with period $\zeta$. 
- $Q_{0}=0, Q_{1}=(1-\zeta) / 3$ and $Q_{2}=-(1-\zeta) / 3$ in $E_{\zeta}$ : the fixed points of the scalar multiplication by $\zeta$ on $E_{\zeta}$.

- $Q_{i_{1} i_{2} \cdots i_{n}}=\left(Q_{i_{1}}, Q_{i_{2}}, \ldots, Q_{i_{n}}\right) \in E_{\zeta}^{n}$ for $i_{1}, i_{2}, \ldots, i_{n} \in\{0,1,2\}$.

- $\bar{Q}_{i_{1} i_{2} \cdots i_{n}}$ is the image of $Q_{i_{1} i_{2} \cdots i_{n}}$ in $E_{\zeta}^{n} /\langle\zeta\rangle$.

- $\bar{X}=E_{\zeta}^{3} /\langle\zeta\rangle, B=E_{\zeta}^{2} /\langle\zeta\rangle$. Here $E_{\zeta}^{2}$ in the definition of $B$ is the product of the first two factors of $E_{\zeta}^{3}$.

- $q: E_{\zeta}^{3} \longrightarrow \bar{X}$ is the quotient map.

- $\operatorname{pr}_{i}: E_{\zeta}^{3} \longrightarrow E_{\zeta}$ is the projection to the $i$ th factor.

- $\operatorname{pr}_{i j}: E_{\zeta}^{3} \longrightarrow E_{\zeta}^{2}$ is the projection to the product of $i$ th and $j$ th factors.

- $p_{i j}: \bar{X} \longrightarrow E_{\zeta}^{2} /\langle\zeta\rangle$ and $p_{i}: \bar{X} \longrightarrow E_{\zeta} /\langle\zeta\rangle$ are the the morphisms induced by $\operatorname{pr}_{i j}$ and $\operatorname{pr}_{i}$, respectively.

- $g_{i}: B \longrightarrow E_{\zeta} /\langle\zeta\rangle=\mathbf{P}^{1}$ is the morphism, induced by the projection $E_{\zeta}^{2} \longrightarrow E_{\zeta}$ to the $i$ th factor $(i=1,2)$.

- $\pi: X_{\phi} \longrightarrow \bar{X}$ is the blow-up at $\left\{\bar{Q}_{i j k} \mid i, j, k=0,1,2\right\}$.

- $p_{\phi}=p_{12} \circ \pi: X_{\phi} \longrightarrow B$.

- $p_{T}: X_{T} \longrightarrow B$ is the projection, induced by $p_{\phi}$.

- $E_{i j k} \simeq \mathbf{P}^{2}$ is the exceptional divisor over $\bar{Q}_{i j k}$ by the blow-up $\pi$ : $X_{\phi} \longrightarrow \bar{X}$.

- $F_{i j k} \simeq \mathbf{F}_{1}$ is the proper transformation of $E_{i j k}$ in $X_{T}$.

The next lemma will be also frequently used in the next two sections:

Lemma 2.1. Let $Z$ be a Calabi-Yau threefold and let $D$ be a smooth divisor on $Z$. Then, $D^{3}=c_{1}\left(T_{D}\right)^{2}$ and $D \cdot c_{2}(Z)=-c_{1}\left(T_{D}\right)^{2}+c_{2}\left(T_{D}\right)$.

We also note that $c_{1}\left(T_{D}\right)^{2}=K_{D}^{2}$ and that $c_{2}\left(T_{D}\right)=c_{2}(D)$ is the topological Euler number of the surface $D$.

Proof. This follows from the fact that $c_{1}(Z)=0$ and the normal sequence

$$
0 \longrightarrow T_{D} \longrightarrow T_{Z} \mid D \longrightarrow N_{Z \mid D} \longrightarrow 0 .
$$




\section{Topological difference between $X_{\phi}$ and $X_{T}$}

In this section we shall prove (0.1) of Theorem 0.1, i.e., that $X_{\phi}$ and $X_{T}$ are not homeomorphic. Since the linear form $c_{2}(Z): H^{2}(Z, \mathbf{Z}) \longrightarrow \mathbf{Z}$ and the cubic form $c_{Z}: \operatorname{Sym}^{3} H^{2}(Z, \mathbf{Z}) \longrightarrow \mathbf{Z}$ are topological invariants, the result follows from:

Theorem 3.1. (1) The linear form given by $c_{2}\left(X_{\phi}\right)$ is divisible by 6 , i.e. $D \cdot c_{2}\left(X_{\phi}\right) \equiv 0(\bmod 6)$ for each $D \in H^{2}\left(X_{\phi}, \mathbf{Z}\right)$, while the linear form $c_{2}\left(X_{T}\right)$ is not.

(2) The cubic form of $X_{\phi}$ is divisible by 3 , i.e., $D^{3} \equiv 0(\bmod 3)$ for each $D \in H^{2}\left(X_{\phi}, \mathbf{Z}\right)$, while the cubic form of $X_{T}$ is not.

For the main result, the statement (2) is sufficient but we also add statement (1) for its own interest.

Remark 3.1. As far as we know, Friedman is the first who found a pair of birational projective Calabi-Yau threefolds which are not homeomorphic [5, Example 7.7]. His examples are based on [17] and they are not rigid. Our proof here is inspired by his argument there.

We shall prove Theorem 3.1 in the sequel.

Let $F \simeq \mathbf{F}_{1}$ be one of $F_{i j k}$ in $X_{T}$. Then, by Lemma 2.1,

$$
F^{3}=K_{F}^{2}=8, F \cdot c_{2}\left(X_{T}\right)=c_{2}(F)-K_{F}^{2}=4-8=-4 .
$$

Clearly, none of them is divisible by 3 .

In the rest of this section, we shall show 6-divisibility of the linear form $c_{2}\left(X_{\phi}\right)$. Three-divisibility of the cubic form then follows from the RiemannRoch formula (cf. Introduction). Here we note that $\operatorname{Pic} X_{\phi} \simeq H^{2}\left(X_{\phi}, \mathbf{Z}\right)$. From now until the end of this section, we write

$$
E=E_{\zeta}, \quad X=X_{\phi} .
$$

For other notations, see Notation 2.1.

Proposition 3.1. (1) The Néron-Severi group $\mathrm{NS}\left(E^{2}\right)$ is generated by the classes of the four divisors, $\{0\} \times E, E \times\{0\}, \Delta$ and $\Gamma$. Here $\Delta$ is the diagonal and $\Gamma$ is the graph of the automorphism $\zeta: E \longrightarrow E$.

(2) The Néron-Severi group $\mathrm{NS}\left(E^{3}\right)$ is generated by the subgroups $\mathrm{pr}_{i j}^{*}$ $\mathrm{NS}\left(E^{2}\right)(1 \leq i<j \leq 3)$. 
Proof. The four classes in (1) are clearly in $\mathrm{NS}\left(E^{2}\right)$, and their intersection matrix is

$$
\left(\begin{array}{llll}
0 & 1 & 1 & 1 \\
1 & 0 & 1 & 1 \\
1 & 1 & 0 & 3 \\
1 & 1 & 3 & 0
\end{array}\right)
$$

The discriminant of this matrix is 3 . On the other hand, the discriminant of the transcendental lattice of $E^{2}$ is 3 by Chioda and Mitani [18]. Thus, the discriminant of $\mathrm{NS}\left(E^{2}\right)$ is also 3. Since $\mathrm{NS}\left(E^{2}\right)$ is torsion free, assertion (1) follows.

Let us show (2). By the Künneth formula, we have

$$
H^{2}\left(E^{3}, \mathbf{Z}\right)=\bigoplus_{i=1}^{3} \operatorname{pr}_{i}^{*} H^{2}(E, \mathbf{Z}) \oplus \bigoplus_{1 \leq i<j \leq 3} \operatorname{pr}_{i j}^{*}\left(H^{1}(E, \mathbf{Z}) \otimes H^{1}(E, \mathbf{Z})\right)
$$

This decomposition is compatible with the Hodge decomposition. Since $\operatorname{NS}\left(E^{3}\right)=H^{2}\left(E^{3}, \mathbf{Z}\right) \cap H^{1,1}\left(E^{3}\right)$ by the Lefschetz $(1,1)$-theorem, we have then

$\mathrm{NS}\left(E^{3}\right)=\bigoplus_{i=1}^{3} \operatorname{pr}_{i}^{*} H^{2}(E, \mathbf{Z}) \oplus \bigoplus_{1 \leq i<j \leq 3} \operatorname{pr}_{i j}^{*}\left(H^{1}(E, \mathbf{Z}) \otimes H^{1}(E, \mathbf{Z}) \cap H^{1,1}\left(E^{2}\right)\right)$.

Again, by the Lefschetz $(1,1)$-theorem, the groups $\operatorname{pr}_{k}^{*} H^{2}(E, \mathbf{Z})(k=i, j)$ and $\operatorname{pr}_{i j}^{*}\left(H^{1}(E, \mathbf{Z}) \otimes H^{1}(E, \mathbf{Z}) \cap H^{1,1}\left(E^{2}\right)\right)$ are subgroups of $\operatorname{pr}_{i j}^{*} \operatorname{NS}\left(E^{2}\right)$, in which $E^{2}$ is the product of $i$ th and $j$ th factors of $E^{3}$. This implies (2).

Recall that $\bar{X}=E^{3} /\langle\zeta\rangle$. In particular, $\bar{X}$ is $\mathbf{Q}$-factorial. A bit more precisely, the divisor $3 D$ is Cartier for any Weil divisor $D$ on $\bar{X}$. Let $N^{1}(\bar{X})$ be the group of the numerically equivalent classes of Weil divisors on $\bar{X}$. Note that Cartier divisors and Weil divisors are the same on $E^{3}$ or on $X$ (as $E^{3}$ and $X$ are smooth) and the numerical equivalence and the algebraic equivalence of divisors are also the same on $E^{3}$ or on $X$ (as their NéronSeveri groups are torsion free).

Proposition 3.2. The group homomorphism $q^{*}: N^{1}(\bar{X}) \longrightarrow \mathrm{NS}\left(E^{3}\right)$ is an isomorphism.

Proof. Our argument here is similar to [12]. Since $\bar{X}$ is $\mathbf{Q}$-factorial and $q$ is finite, the group homomorphism $q^{*}$ is indeed well defined and injective.

Let $[H] \in \mathrm{NS}\left(E^{3}\right)$. We need to find $D \in N^{1}(\bar{X})$ such that $[H]=q^{*} D$. 
Claim 1. We can (and will) choose the representative $H \in \mathrm{Pic} E^{3}$ of the class $[H]$, such that $\zeta^{*} H=H$ as line bundles.

Proof. Take the origin of $E$ as polarization of $E$. One can then identify $\operatorname{Pic}^{0}(E)=E$ in an equivariant way with respect to the action of $\zeta$. Under this identification, we have an identification $\operatorname{Pic}^{0}\left(E^{3}\right)=E^{3}$ in which the action of $\zeta^{*}$ on $\operatorname{Pic}^{0}\left(E^{3}\right)$ is the same as the diagonal action $(\zeta, \zeta, \zeta)$ on $E^{3}$. Note also that $\zeta^{*}=$ id on $\mathrm{NS}\left(E^{3}\right)$ as $\zeta^{*}=$ id on the wider space $H^{1,1}\left(E^{3}\right)$.

Put $T=\zeta^{*} H-H$. Here the equality is as line bundles. Then $T=\left(T_{1}, T_{2}\right.$, $\left.T_{3}\right)$ is an element of $\operatorname{Pic}^{0}\left(E^{3}\right)=E^{3}$, as $\zeta^{*}[H]=[H]$. Note that there is a point $P=\left(P_{1}, P_{2}, P_{3}\right) \in \operatorname{Pic}^{0}\left(E^{3}\right)=E^{3}$ such that

$$
\left(P_{1}, P_{2}, P_{3}\right)-\left(\zeta P_{1}, \zeta P_{2}, \zeta P_{3}\right)=\left(T_{1}, T_{2}, T_{3}\right)
$$

The line bundle $H+P$ is a desired representative.

From now, we regard $H$ as an effective divisor in $|H|$ rather than the line bundle.

Claim 2. We may (and will) assume that there is an effective divisor $H$ in $|H|$ such that $\zeta^{*} H=H$ as divisors.

Proof. Since $q$ is finite, the divisor $q^{*} A$ is ample if $A$ is ample. Thus, by adding $q^{*} A$ with sufficiently ample $A$ to $H$, we may assume that $|H|$ is a free linear system. Since $\zeta^{*} H=H$ as line bundles, $\zeta$ acts on the projective space $|H|$. This action certainly has a fixed points. Let $H$ be a divisor corresponding to (one of) the fixed point. Then $\zeta^{*} H=H$ as divisors on $E^{3}$.

Let $\bar{H}=q_{*} H$ as Weil divisors. Since $\zeta^{*} H=H$ as divisors and $\left(E^{3}\right)^{\langle\zeta\rangle}$ consists of finitely many points, there is a divisor $D$ such that $\bar{H}=3 D$ as Weil divisors. For this $D$, we have

$$
3 q^{*} D=q^{*} \bar{H}=H+\zeta^{*} H+\left(\zeta^{*}\right)^{2} H=3 H .
$$

Since $\operatorname{NS}\left(E^{3}\right)$ is torsion free, this implies $q^{*} D=H$.

Proposition 3.3. Let $\tilde{D}_{i j l}(1 \leq l \leq 4)$ be the divisors on $E^{3}$, which are pull back of the four divisors $E \times\{0\},\{0\} \times E, \Delta$ and $\Gamma$ on $E^{2}$ by $p_{i j}(1 \leq i<$ $j \leq 3)$. Let $\bar{D}_{i j l}:=\left(q_{*} \tilde{D}_{i j l}\right)_{\text {red. }}$. Then, the (classes of) 12 Weil divisors $\bar{D}_{i j l}$ generate $N^{1}(\bar{X})$. 
Proof. We note that $\zeta^{*} \tilde{D}_{i j l}=\tilde{D}_{i j l}$ as divisors on $E^{3}$. Thus $\tilde{D}_{i j l}=\pi^{*} \bar{D}_{i j l}$ (cf. Proof of Proposition 3.2). Since $\tilde{D}_{i j l}$ generate $\mathrm{NS}\left(E^{3}\right)$ by Proposition 3.1, the result follows from Proposition 3.2.

Let $D_{i j l}$ be the proper transform of $\bar{D}_{i j l}$ on $X$ by $\pi: X \longrightarrow \bar{X}$.

Proposition 3.4. $\mathrm{NS}(X)$ is contained in the subgroup of $\mathrm{NS}(X) \otimes \mathbf{Q}$ generated by the classes of the following divisors:

$$
D_{i j l}, E_{i j k}, T_{\Lambda, \Lambda^{\prime}}:=\frac{1}{3} \sum_{(i, j, k) \in \Lambda} E_{i j k}+\frac{1}{3} \sum_{\left(i^{\prime}, j^{\prime}, k^{\prime}\right) \in \Lambda^{\prime}} 2 E_{i^{\prime} j^{\prime} k^{\prime}}
$$

where $\Lambda$ and $\Lambda^{\prime}$ are some disjoint subsets (possibly empty) of the product set $\{0,1,2\}^{3}$ such that $\Lambda \cap \Lambda^{\prime}=\emptyset$ and such that both $|\Lambda|$ and $\left|\Lambda^{\prime}\right|$ are divisible by 3 .

Proof. Let $D$ be a prime divisor on $X$. Put $\bar{D}=\pi_{*} D$ as Weil divisors. Then, by Proposition 3.3, there are integers $b_{i j l}$ such that $\bar{D}=\sum_{i, j, l} b_{i j l} \bar{D}_{i j l}$ in $N^{1}(\bar{X})$. Since $3 \bar{D}$ and $3 \bar{D}_{i j l}$ are Cartier, there are integers $a_{i j k}$ such that

$$
D=\sum_{i, j, l} b_{i j l} D_{i j l}+\frac{1}{3} \sum_{i, j, k} a_{i j k} E_{i j k}
$$

in $\mathrm{NS}(X) \simeq \operatorname{Pic} X$. So, the result follows from the next lemma.

Lemma 3.1. Let $\Lambda$ and $\Lambda^{\prime}$ be subset of $\{0,1,2\}^{3}$ such that $\Lambda \cap \Lambda^{\prime}=\emptyset$. If

$$
M:=\sum_{(i, j, k) \in \Lambda} E_{i j k}+\sum_{\left(i^{\prime}, j^{\prime}, k^{\prime}\right) \in \Lambda^{\prime}} 2 E_{i^{\prime} j^{\prime} k^{\prime}}
$$

is divisible by 3 in $\operatorname{Pic} X$, then both $|\Lambda|$ and $\left|\Lambda^{\prime}\right|$ are divisible by 3 .

Proof. Let $\alpha \in\{0,1,2\}$. Let $D_{\alpha}$ be the divisor on $X$, which is the proper transform of the divisor $\bar{D}_{\alpha}=\left(p_{3}^{*} Q_{\alpha}\right)_{\text {red }}$ on $\bar{X}$ (See figure 3 and Notation 2.1 for $\left.Q_{\alpha}\right)$.

Since $\bar{D}_{\alpha}$ passes through 9-singular points of $\bar{X}$, the surface $D_{\alpha}$ meets the 9-exceptional divisors, say,

$$
E_{00 \alpha}, E_{01 \alpha}, E_{02 \alpha}, E_{10 \alpha}, E_{11 \alpha}, E_{12 \alpha}, E_{20 \alpha}, E_{21 \alpha}, E_{22 \alpha}
$$

We put $l_{i j \alpha}:=\left.E_{i j \alpha}\right|_{D_{\alpha}}$. These are all $(-3)$-curves. The surface $D_{\alpha}$ is a nonrelatively minimal rational elliptic surface with 3 -singular fibers $l_{i 0 \alpha}+l_{i 1 \alpha}+$ 


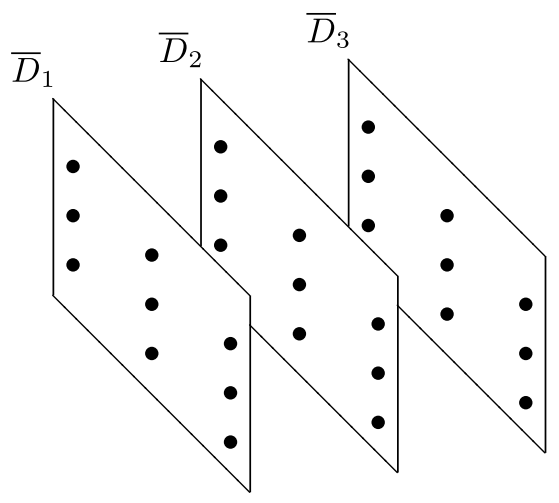

Figure 3: $D_{\alpha}$ 's

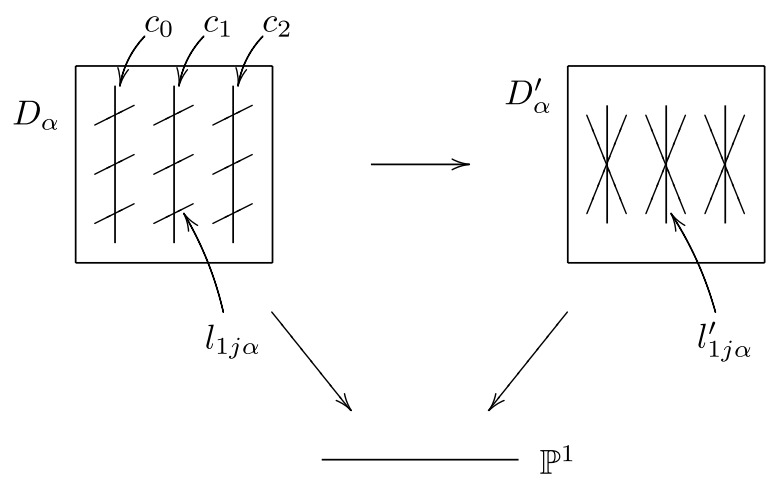

Figure 4: $\nu_{\alpha}: D_{\alpha} \rightarrow D_{\alpha}^{\prime}$

$l_{i 2 \alpha}+3 c_{i}(i=0,1,2)$ as in the figure below (figure 4). Here $c_{i}$ are $(-1)$ curves. Let $\nu_{\alpha}: D_{\alpha} \longrightarrow D_{\alpha}^{\prime}$ be the contraction of the three $(-1)$-curves $c_{i}$. Let $l_{i j \alpha}^{\prime}=\nu\left(l_{i j \alpha}\right)$. Then $D_{\alpha}^{\prime}$ is a relatively minimal rational elliptic surface with 3 singular fibers $l_{i 0 \alpha}^{\prime}+l_{i 1 \alpha}^{\prime}+l_{i 2 \alpha}^{\prime}$ (figure 4 ). Since $M$ is 3 -divisible, so is the divisor

$$
M_{\alpha}^{\prime}:=\left(\nu_{\alpha}\right)_{*}\left(\left.M\right|_{D_{\alpha}}\right)=\sum_{i, j} a_{i j \alpha} l_{i j \alpha}^{\prime} .
$$

Our $D_{\alpha}^{\prime}$ belongs to No.39 in the list of [15]. In particular, the MordellWeil group has a torsion element of order 3 . Thus, there are three sections $s_{0}, s_{1}$ and $s_{2}$ which meet $l_{00 \alpha}^{\prime}, l_{01 \alpha}^{\prime}, l_{02 \alpha}^{\prime}$, respectively. On the other hand, since $M_{\alpha}^{\prime} \cdot l_{i j \alpha}^{\prime}$ are divisible by 3 , the set of three elements $\left\{a_{i 0 \alpha}, a_{i 1 \alpha}, a_{i 2 \alpha}\right\}$ (counted with multiplicities) is either one of $\{0,0,0\},\{1,1,1\},\{2,2,2\}$, $\{0,1,2\}$, for each $i=0,1,2$. Suppose that for $i=0$ we have $\left\{a_{00 \alpha}, a_{01 \alpha}\right.$, 
$\left.a_{02 \alpha}\right\}=\{0,1,2\}$. Then, the same holds for $i=1$ and 2 . This is because $s_{0}$. $M_{\alpha}^{\prime}, s_{1} \cdot M_{\alpha}^{\prime}$ and $s_{2} \cdot M_{\alpha}^{\prime}$ are all divisible by 3 . Thus both $\mid \Lambda \cap\{(i, j, \alpha) \mid i, j=$ $0,1,2\} \mid$ and $\left|\Lambda^{\prime} \cap\{(i, j, \alpha) \mid i, j=0,1,2\}\right|$ are divisible by 3 for each $\alpha \in$ $\{0,1,2\}$. This implies the result.

Now we are ready to prove 6-divisibility of the linear from $c_{2}(X)$. It suffices to check that $D \cdot c_{2}(X) \equiv 0 \bmod 6$ for $D_{i j l}, E_{i j k}$ and $T_{\Lambda, \Lambda^{\prime}}$ in Proposition 3.4 .

We have $K_{E_{i j k}}^{2}=E_{i j k}^{3}=9$ and $c_{2}\left(E_{i j k}\right)=3$, as $E_{i j k} \simeq \mathbf{P}^{2}$. Thus $E_{i j k}$. $c_{2}(X)=-6$ by Lemma 2.1. This also implies 6-divisibility of $T_{\Lambda, \Lambda^{\prime}} \cdot c_{2}(X)$ as both $|\Lambda|$ and $\left|\Lambda^{\prime}\right|$ are divisible by 3 .

Let us compute $D_{i j l} \cdot c_{2}(X)$. As we have observed in Lemma 3.1, the surface $D_{i j l}$ is the blow up at three points of a relatively minimal rational elliptic surface. Thus, $K_{D_{i j l}}^{2}=-3$ and $c_{2}\left(D_{i j l}\right)=15$, and therefore, $D_{i j l}$. $c_{2}(X)=18$ by Lemma 2.1 .

This completes the proof Theorem 3.1.

\section{Connecting $X_{\phi}$ and $X_{T}$ by projective flat deformation}

In this section we shall prove (0.1) in Theorem 0.1, i.e., $X_{\phi}$ and $X_{T}$ are connected by projective flat deformation. By Theorem 0.3, this follows from:

Theorem 4.1. There are ample divisors $H_{\phi}$ on $X_{\phi}$ and $H_{T}$ on $X_{T}$ such that

$$
H_{\phi} \cdot c_{2}\left(X_{\phi}\right)=H_{T} \cdot c_{2}\left(X_{T}\right) \text { and } H_{\phi}^{3}=H_{T}^{3} .
$$

We shall prove Theorem 4.1 in the sequel. In the proof, we freely use the notations given in Notation 2.1.

\subsection{Construction of a divisor $H_{\phi}$ on $X_{\phi}$}

Recall that $E_{\zeta} /\langle\zeta\rangle \simeq \mathbb{P}^{1}$. Let $\bar{L}_{i}=p_{i}^{*} \mathcal{O}_{E_{\zeta} /\langle\zeta\rangle}(1)$ and $L_{i}=\pi^{*} \bar{L}_{i}$. Let

$$
H_{\phi}=-\sum_{i, j, k} E_{i j k}+x L_{1}+y L_{2}+z L_{3}
$$

where $x, y$ and $z$ are positive integers.

Lemma 4.1. (1) For sufficiently large number $C, H_{\phi}$ is ample on $X_{\phi}$ when $x>C, y>C, z>C$. 
(2) $H_{\phi} \cdot c_{2}\left(X_{\phi}\right)=162$.

(3) $H_{\phi}^{3}=54 x y z-243$.

Proof. By construction, the divisor $-\sum_{i, j, k} E_{i j k}$ is $\pi$-ample, the divisors $\bar{L}_{i}$ 's are nef on $\bar{X}$ and $\bar{L}_{1}+\bar{L}_{2}+\bar{L}_{3}$ is ample on $\bar{X}$. This implies (1). Note that $L_{i}$ is represented by a smooth abelian surface and $E_{i j k} \simeq \mathbf{P}^{2}$. Thus, by Lemma 2.1 , we have $L_{i} \cdot c_{2}\left(X_{\phi}\right)=0$ and $E_{i j k} \cdot c_{2}\left(X_{\phi}\right)=-6$. This implies (2). Note also that

$$
E_{i j k}^{3}=9, L_{1} \cdot L_{2} \cdot L_{3}=9, E_{i j k} \cdot L_{l}=L_{i}^{2}=0
$$

and $E_{i j k} \cdot E_{l m n}=0$ unless $(i, j, k)=(l, m, n)$. Therefore we have

$$
\begin{aligned}
H_{\phi}^{3}= & \left(-\sum_{i, j, k} E_{i j k}\right)^{3}+3\left(-\sum_{i, j, k} E_{i j k}\right)^{2}\left(x L_{1}+y L_{2}+z L_{3}\right) \\
& +3\left(-\sum_{i, j, k} E_{i j k}\right)\left(x L_{1}+y L_{2}+z L_{3}\right)^{2} \\
& +\left(x L_{1}+y L_{2}+z L_{3}\right)^{3} \\
= & -\sum_{i, j, k} E_{i j k}^{3}+0+0+6 x y z L_{1} \cdot L_{2} \cdot L_{3} \\
= & 54 x y z-243 .
\end{aligned}
$$

\subsection{Construction of a divisor $H_{T}$ on $X_{T}$}

We recall the following commutative diagram:

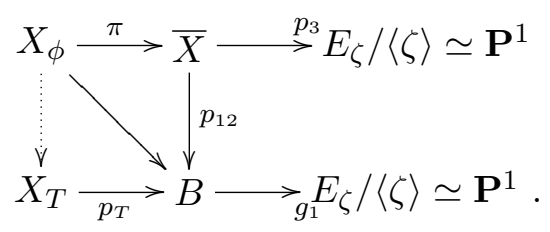

Let $l_{i}^{\prime}=g_{1}^{-1}\left(\bar{Q}_{i}\right)$ and $\left.M_{i}=\overline{p_{T}^{-1}\left(l_{i}^{\prime} \backslash\left\{\bar{Q}_{i 0}, \bar{Q}_{i 1}, \bar{Q}_{i 2}\right\}\right.}\right)(i=0,1,2)$. Then $M_{i}$ is a relatively minimal rational elliptic surface. We denote a general smooth fiber of the fibration $M_{i} \longrightarrow \mathbf{P}^{1}$ by $f_{M_{i}}$. By construction, $M_{i}$ has 3 


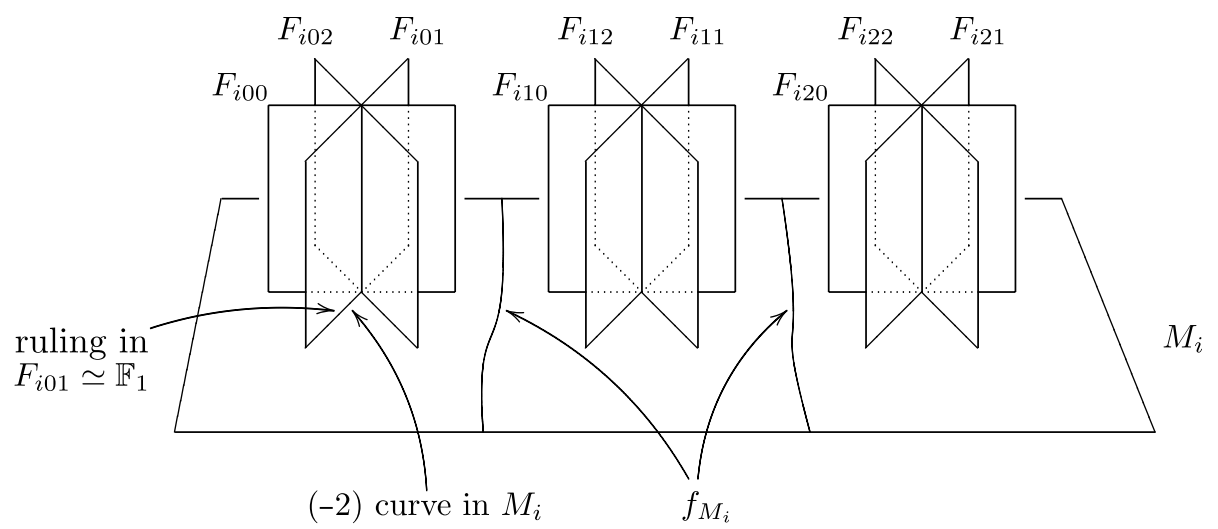

Figure 5: $M_{i}$ and $F_{i j k}$ 's in $X_{T}$

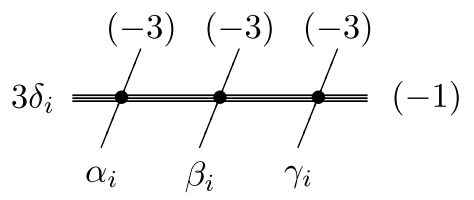

Figure 6: $\eta_{i}$

singular fibers of Kodaira type $I V$ :

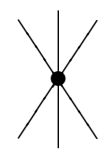

See figure 5 for $M_{i}$ and the way $M_{i}$ intersects with $F_{i j k}$ 's.

Let $\bar{S}_{j}=\left(p_{3}^{*}\left(\bar{Q}_{j}\right)\right)_{\text {red }}$ on $\bar{X}$ and $S_{j}$ be the proper transformation of $\bar{S}_{j}$ on $X_{T}(j=0,1,2)$. Then $S_{j}$ is a (non-relatively minimal) rational elliptic surface with three singular fibers (denote them by $\eta_{1}, \eta_{2}$ and $\eta_{3}$ ) that are composed of one $(-1)$-curve of multiplicity 3 and three $(-3)$-curves; $\eta_{i}=$ $\alpha_{i}+\beta_{i}+\gamma_{i}+3 \delta_{i}$. See figure 6 . We denote by $f_{S_{j}}$ a general smooth fiber of the fibration $S_{j} \longrightarrow \mathbf{P}^{1}$.

See figure 7 for the configuration of $S_{j}, M_{i}$ and $F_{\alpha \beta \gamma}$ 's.

Lemma 4.2. The following divisor is $p_{T}$-ample;

$$
3\left(M_{0}+M_{1}+M_{2}\right)+S_{0}+S_{1}+S_{2}
$$




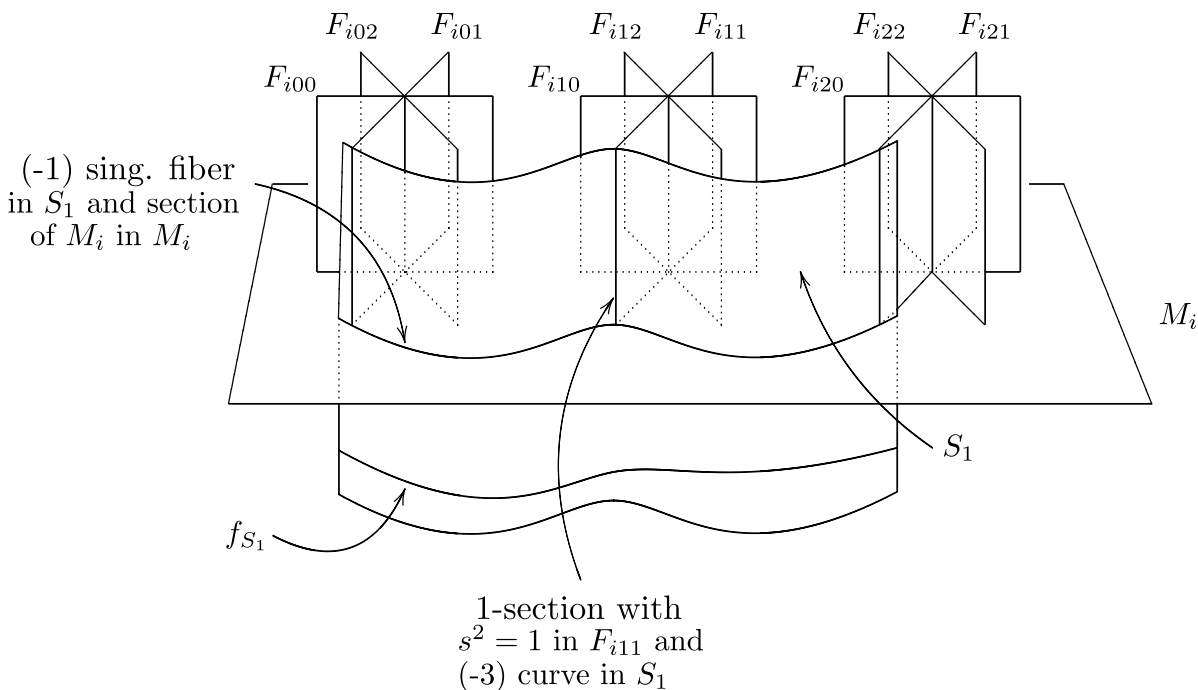

Figure 7: $M_{i}, F_{i j k}$ 's and $S_{1}$ in $X_{T}$

Proof. Since $S_{j}$ are sections of $p_{T}$ over $B \backslash\left\{\bar{Q}_{\alpha \beta} \mid \alpha, \beta=0,1,2\right\}$, we only need to check that $3\left(M_{0}+M_{1}+M_{2}\right)+S_{0}+S_{1}+S_{2}$ is ample on $F_{\alpha \beta \gamma}$ 's. This, however, follows from the fact that

$$
\left.\left(3\left(M_{0}+M_{1}+M_{2}\right)+S_{0}+S_{1}+S_{2}\right)\right)\left.\right|_{F_{\alpha \beta \gamma}}=3 f+s
$$

where $f$ is the ruling of the ruled surface $F_{\alpha \beta \gamma} \simeq \mathbf{F}_{1}$ and $s$ is a positive section (with $s^{2}=1$ ) (see figures 5 and 7 ).

Let $\bar{A}_{k}$ be a general fiber of $g_{k}: B \longrightarrow E_{\zeta} /\langle\zeta\rangle$ and let $A_{k}=p_{T}^{*} \bar{A}_{k}(k=1$, 2). $\bar{A}_{k}$ is an elliptic curve and $A_{k}$ is an abelian surface. Note that $\bar{A}_{k} \in$ $\left|g_{k}^{*} \mathcal{O}_{E_{\zeta} /\langle\zeta\rangle}(1)\right|$. Put

$$
H_{T}:=3\left(M_{0}+M_{1}+M_{2}\right)+S_{0}+S_{1}+S_{2}+a A_{1}+b A_{2} .
$$

Here $a$ and $b$ are positive integers.

Lemma 4.3. (1) For sufficiently large number $C, H_{T}$ is ample on $X_{T}$ when $a>C, b>C$.

(2) $H_{T} \cdot c_{2}\left(X_{T}\right)=162$.

(3) $H_{T}^{3}=18 a b-27 b-333$. 
Proof. Since $\bar{A}_{1}, \bar{A}_{2}$ are nef and $\bar{A}_{1}+\bar{A}_{2}$ is an ample on $B$, the first assertion follows from Lemma 4.2. By using Lemma 2.1, we compute that

$$
\begin{aligned}
M_{i} \cdot c_{2}\left(X_{T}\right) & =-K_{M_{i}}^{2}+c_{2}\left(M_{i}\right)=12 \\
S_{j} \cdot c_{2}\left(X_{T}\right) & =-K_{S_{j}}^{2}+c_{2}\left(S_{j}\right)=18 \\
A_{k} \cdot c_{2}\left(X_{T}\right) & =-K_{A_{k}}^{2}+c_{2}\left(A_{k}\right)=0 .
\end{aligned}
$$

This implies the second assertion. For the third one, we first expand $H_{T}^{3}$ as

$$
\begin{array}{rlrl}
H_{T}^{3}= & \left(3\left(M_{0}+M_{1}+M_{2}\right)+S_{0}+S_{1}+S_{2}\right)^{3} & & \left(=Q_{1}\right) \\
& +\left(3\left(M_{0}+M_{1}+M_{2}\right)+S_{0}+S_{1}+S_{2}\right)^{2}\left(a A_{1}+b A_{2}\right) & & \left(=Q_{2}\right) \\
& +\left(3\left(M_{0}+M_{1}+M_{2}\right)+S_{0}+S_{1}+S_{2}\right)\left(a A_{1}+b A_{2}\right)^{2} & & \left(=Q_{3}\right) \\
& +\left(a A_{1}+b A_{2}\right)^{3} & & \left(=Q_{4}\right) \\
= & Q_{1}+Q_{2}+Q_{3}+Q_{4} . &
\end{array}
$$

We compute $Q_{1}, Q_{2}, Q_{3}, Q_{4}$ separately.

$Q_{1}$ : Note that

$$
\begin{aligned}
& S_{j}^{3}=K_{S_{j}}^{2}=-3 \\
& M_{i}^{3}=K_{M_{i}}^{2}=0 \\
& S_{i} \cdot S_{j}=M_{i} \cdot M_{j}=0 \text { for } i \neq j \\
& M_{i}^{2} \cdot S_{j}=\left(\left.M_{i}\right|_{S_{j}}\right)^{2}=-1 \\
& M_{i} \cdot S_{j}^{2}=\left(\left.S_{j}\right|_{M_{i}}\right)^{2}=-1 .
\end{aligned}
$$

With these, we have $Q_{1}=-333$.

$Q_{2}$ : We observe that

$$
-\left.M_{i}\right|_{M_{i}} \sim-\left.K_{M_{i}} \sim A_{1}\right|_{M_{i}} \sim f_{M_{i}} \quad \text { and } \quad f_{M_{i}} \cdot A_{2}=3
$$

From this we have $M_{i}^{2} \cdot\left(a A_{1}+b A_{2}\right)=-3 b$. Note also that

$$
\left.A_{1}\right|_{S_{j}} \sim f_{S_{j}}
$$

It follows that

$$
\begin{aligned}
M_{i} \cdot S_{j} \cdot\left(a A_{1}+b A_{2}\right) & =\left.\left.M_{i}\right|_{S_{j}} \cdot\left(a A_{1}+b A_{2}\right)\right|_{S_{j}} \\
& =b\left(\delta_{i} \cdot A_{2}\right) \\
& =b .
\end{aligned}
$$


See also figure 6. Finally,

$$
\begin{aligned}
S_{j}^{2} \cdot\left(a A_{1}+b A_{2}\right) & =\left.K_{S_{j}} \cdot\left(a A_{1}+b A_{2}\right)\right|_{S_{j}} \\
& =b\left(-f_{S_{j}}+\delta_{1}+\delta_{2}+\delta_{3}\right) \cdot A_{2} \\
& =b(-3+1+1+1) \\
& =0 .
\end{aligned}
$$

Thus we have $Q_{2}=-27 b$.

$Q_{3}$ : Note that $\left(a A_{1}+b A_{2}\right)^{2}=6 a b\left(\right.$ fiber of $\left.p_{T}\right)$. So we have

$$
\begin{aligned}
& M_{i} \cdot\left(a A_{1}+b A_{2}\right)^{2}=0 \\
& S_{j} \cdot\left(a A_{1}+b A_{2}\right)^{2}=6 a b
\end{aligned}
$$

Thus, $Q_{3}=18 a b$.

$Q_{4}:$ Clearly, $Q_{4}=0$.

With all these, we obtain $H_{T}^{3}=Q_{1}+Q_{2}+Q_{3}+Q_{4}=18 a b-27 b-333$.

\subsection{Synthesis}

Now we are ready to prove Theorem 4.1. By Lemmas 4.1 and 4.3, the divisors $H_{\phi}$ and $H_{T}$ are ample on $X_{\phi}$ and $X_{T}$, respectively, when $x, y, z$ and $a, b$ are greater than some sufficiently large $C$. So, it suffices to find integers $x, y, z$ and $a, b$ greater than any given positive integer $C$ that satisfy the following equations:

$$
162=H_{\phi} \cdot c_{2}\left(X_{\phi}\right)=H_{T} \cdot c_{2}\left(X_{T}\right)=162
$$

and

$$
54 x y z-243=H_{\phi}^{3}=H_{T}^{3}=18 a b-27 b-333 .
$$

The first one poses no condition on $x, y, z, a, b$, and the second one is simplified to

$$
6 x y z=2 a b-3 b-10
$$

For a given positive integer $C$, let

$$
x=12 C^{2}-6, y=z=2 C, a=6 C^{2}+1, b=24 C^{2}-10 .
$$

Then $x, y, z$ and $a, b$ are integers which are greater than $C$ and satisfy the above Equation (1).

This completes the proof of Theorem 4.1. 


\section{Acknowledgment}

We would like to express our thanks to Professors J.M. Hwang, J.H. Keum and B. Kim for valuable discussions. The anonymous referees also gave several valuable suggestions. K.O. was supported by JSPS.

\section{References}

[1] V. Batyrev, Birational Calabi-Yau n-folds have equal Betti numbers, (English summary) New trends in algebraic geometry (Warwick, 1996), London Math. Soc. Lecture Note Ser., 264, Cambridge Univ. Press, Cambridge, 1999, 1-11.

[2] A. Beauville, Variétés Kähleriennes dont la premiére classe de Chern est nulle, J. Differential Geom. 18 (1983), 755-782.

[3] A. Beauville, Some remarks on Kähler manifolds with $c_{1}=0$, Classification of algebraic and analytic manifolds (Katata, 1982), Progr. Math., 39, Birkhauser Boston, Boston, MA, 1983, 1-26.

[4] J. Denef and F. Loeser, Germs of arcs on singular algebraic varieties and motivic integration, Invent. Math. 135 (1999), 201-237.

[5] R. Friedman, On threefolds with trivial canonical bundle, Complex geometry and Lie theory (Sundance, UT, 1989), Proc. Sympos. Pure Math., 53, Amer. Math. Soc., Providence, RI, 1991, 103-134.

[6] M. Gross, D. Huybrechts and D. Joyce, Calabi-Yau manifolds and related geometries, Springer-Verlag, 2003.

[7] R. Hartshorne, Connectedness of the Hilbert scheme, Inst. Hautes Etudes Sci. Publ. Math. No. 29 (1966), 5-48.

[8] D. Huybrechts, Compact hyperkähler manifolds: basic results, Invent. Math. 135 (1999), 63-113; Erratum: Compact hyper-Kähler manifolds: basic results, Invent. Math. 152 (2003), 209-212.

[9] D. Huybrechts, The Kähler cone of a compact hyperkähler manifold, Math. Ann. 326 (2003), 499-513.

[10] T. Ito, Birational smooth minimal models have equal Hodge numbers in all dimensions, in Calabi-Yau varieties and mirror symmetry, eds. N. Yui and J.D. Lewis (Toronto, ON, 2001), Fields Inst. Commun. 38 Amer. Math. Soc., Providence, RI, 2003, 183-194. 
[11] N.-H. Lee, Calabi-Yau construction by smoothing normal crossing varieties, math.AG/0604596.

[12] Y. Namikawa, Periods of Enriques surfaces, Math. Ann. 270 (1985), 201-222.

[13] K. Oguiso, On certain rigid fibered Calabi-Yau threefolds, Math. Z. 221 (1996), 437-448.

[14] K. Oguiso, T. Peternell, On polarized canonical Calabi-Yau threefolds, Math. Ann. 301 (1995), 237-248.

[15] K. Oguiso, T. Shioda, The Mordell-Weil lattice of a rational elliptic surface, Comment. Math. Univ. St. Paul. 40 (1991), 83-99.

[16] I. Peeva, M. Stillman, Connectedness of Hilbert schemes, J. Algebraic Geom. 14 (2005), 193-211.

[17] C. Schoen, On fiber products of rational elliptic surfaces with section, Math. Z. 197 (1988), 177-199.

[18] T. Shioda, N. Mitani, Singular abelian surfaces and binary quadratic forms, in Classification of algebraic varieties and compact complex manifolds, ed. H. Popp, Lecture Notes in Math., 412, 1974, 259-287.

[19] C.-L. Wang, Cohomology theory in birational geometry, J. Differential Geom. 60 (2002), 345-354.

[20] C.-L. Wang, Quasi-Hodge metrics and canonical singularities, Math. Res. Lett. 10 (2003), 57-70.

[21] T. Yasuda, Twisted jets, motivic measures and orbifold cohomology, Compos. Math. 140 (2004), 396-422.

Department of Mathematics Education

HONGIK UNIVERSITY 42-1

SANGSU-Dong, MAPO-GU

SEOUL 121-791

Korea

School of Mathematics

Korea Institute for Advanced Study

DongDaEMUN-GU

SEOUL 130-722

KoreA

E-mail address: nhlee@hongik.ac.kr 
Department of Mathematics

OSAKA UNIVERSITY

TOYONAKA 560-0043

OSAKA

JAPAN

DePartment of ECONOMiCS

KeIO UNIVERSITY

HIYOSHI KOHOKU-KU

YOKOHAMA

JAPAN

School of Mathematics

Korea Institute for Advanced Study

DONGDAEMUN-GU

SEOUL 130-722

KOREA

E-mail address: oguiso@hc.cc.keio.ac.jp

Received OCtober 15, 2008 
